Derechos reservados de El Colegio de Sonora, ISSN 1870-3925

\title{
Las maquiladoras frente al VIH/sida, implementación de programas y percepción de los empleadores en Puebla
}

\author{
Blanca Pelcastre-Villafuerte* \\ Tamil Kendall** \\ Carlos Magis***
}

Resumen: ${ }^{1}$ En la industria maquiladora prevalece la vulnerabilidad social, la violación a los derechos humanos, ciertos estigmas y la discriminación. El presente estudio se realizó en el estado de Puebla, debido al desarrollo de sus maquiladoras y a la tasa relativamente alta de casos de sida, registrada entre las mujeres. Se verificó la existencia de acciones y programas, para enfrentar la epidemia en los sitios de trabajo. Se aplicó un cuestionario a empleadores y entrevistas semiestructuradas a profesionales de la salud, activistas de organizaciones no gubernamentales (ONG) y académicos; se llevó a cabo observación no participante. Las condiciones laborales, en particular de las empresas que operan de forma clandestina, según el esquema de subcontratación e incumplen con lo establecido en la Ley Federal del Trabajo, contribuyen a incrementar la vulnerabilidad ante el VIH/sida de las mujeres trabajadoras. Se evidencia una falta de acciones y programas de prevención de

\footnotetext{
* Doctora en psicología social. Dirección de Salud Comunitaria y Bienestar Social. Centro de Investigación en Sistemas de Salud. Instituto Nacional de Salud Pública.

** Maestra en comunicaciones. Consultora en el virus de inmunodeficiencia humana (VIH), Fondo de Población de las Naciones Unidas (UNFPA, por sus siglas en inglés)-México. En el momento del estudio fue investigadora asociada del Centro Colaborador del Programa Conjunto de las Naciones Unidas dedicado al vIH/sida, ONUSIDA (UNAIDS, por sus siglas en inglés), Centro de Investigación en Sistemas de Salud. Instituto Nacional de Salud Pública. Correspondencia: Balance, A. C. Huatusco 39, Roma Sur, C. P. 06760. México, Distrito Federal.Teléfono: (55) 52-64-33-31.Correo electrónico: tamilhiv@gmail.com

*** Doctor en salud pública. Director de Investigación del Centro Nacional para la Prevención y el Control del vIH/sida (CENSIDA).

${ }^{1}$ Estudio financiado por UNFPA, a través de CENSIDA.
} 
VIH/sida, así como el predominio de estigmas y discriminación entre los empleadores.

Palabras clave: maquila, vIH/sida, vulnerabilidad, lugar de trabajo, México.

Abstract: Social vulnerability, human rights violations, some stigmas and discrimination prevail in the maquiladora industry. The present research was carried out in Puebla, because of the development of the maquiladora industry there, as well as the high rate of AIDS cases among women. It also sought to document the existence of programs which respond to the HIV epidemic in the workplace. Methods included a questionnaire completed by employers and semi-structured interviews with health professionals, activists of non-government organizations (NGO), and academics; nonparticipant observation was also carried out. Prevalent labor conditions, particularly in clandestine maquiladoras that work as sub-contractors, and the failure to comply with federal labor legislation, contribute to raise female workers' vulnerability to HIV and AIDS. The study documented a lack of HIV/AIDS prevention programs in the workplace, as well as stigmatizing attitudes and discrimination among employers.

Key words: maquiladora industry, HIV/AIDS, vulnerability, workplace, Mexico.

\section{Introducción}

La epidemia de sida en el mundo alcanza cifras alarmantes, para finales de 2006 se registraron 39.5 millones de personas infectadas por el VIH, 25 millones eran trabajadores en edad productiva, de 15 a 49 años. En América Latina se calcula una prevalencia de 0.4 por ciento de mujeres y 0.6 de hombres entre 15 y 24 años, que viven con VIH; en México es de 0.3 por ciento en adultos de 15 a 49 años; son alrededor de 180 mil las personas que viven con el VIH (PVVs), de las cuales 42 mil son mujeres (UNAIDS 2007).

Ante este panorama, al igual que las escuelas, el lugar de trabajo es un espacio importante para la educación sexual y para llevar a cabo acciones preventivas, algunas de ellas enfocadas al VIH y a infecciones de trasmisión sexual (ITS). El VIH/sida perjudica el ámbito laboral, pues es en el sector más productivo de la población económicamente activa donde se observa el mayor peso de la enfermedad, en este sentido reduce los ingresos e impone costos ele- 
vados a las empresas, como consecuencia de la disminución de la productividad y la pérdida de trabajadores calificados y con experiencia (Oficina Internacional del Trabajo, oIт 2001). Además, el estigma, entendido como el proceso social de subvaloración generado a partir de un juicio adverso sobre una persona y la discriminación a la que con frecuencia conduce, puede tener repercusiones negativas sobre el acceso al empleo, al propiciar la violación de los derechos humanos de las pvvs (ONUSIDA 2002a). En la Declaración de compromiso en la lucha contra el sida (UNGASS, por sus siglas en inglés), elaborada en un periodo extraordinario de sesiones de la Asamblea General de las Naciones Unidas, en Nueva York, en junio de 2001, se identificó el lugar de trabajo como un sitio imprescindible para emprender acciones y monitorear la respuesta nacional al viH/sida (Naciones Unidas 2001). Los gobiernos internacionales, incluso el mexicano, firmaron esta declaración en la que se comprometieron a crear, para 2003, leyes y políticas que protegieran en el lugar de trabajo los derechos de las PVvs, las que están enfermas o tienen un alto riesgo de contraer el virus (párrafo 69) y, para 2005, también poner en práctica ahí programas de prevención y atención, para proporcionarles un entorno que las apoye (párrafo 49) (ONUSIDA 2002b, 9, 17).

En esta lucha contra el viH/sida, el lugar de trabajo es importante no sólo por influir en la productividad, sino que al constituir el centro integrador de la vida local, representa una posibilidad y un compromiso de evitar la expansión y efectos de la epidemia (OIT 2001, 3; International Labour Organization-AIDS 2004), sin embargo, hasta 2005 México no contaba con programas ni políticas vigentes para enfrentar la epidemia en el lugar de trabajo (UNAIDS 2006, 552).

\section{Vulnerabilidad social y maquila}

La industria maquiladora es un espacio de trabajo donde confluyen un conjunto de condiciones que se traducen en riesgos diferenciales para los empleados, hombres y mujeres frente al vIH/sida. La maquila de exportación o internacional es un sistema de producción, en general bajo la forma de subcontratación, en el que se transforman insumos intermedios y materias primas importadas, y cuyos productos finales se comercializan en el exterior, este modelo se distingue de otro más doméstico, conocido como maquiladora domiciliaria, ubicada en talleres pequeños instalados en los hogares, donde se fabrican, por lo general, prendas de vestir y se sostienen con una inversión nacional, aunque no exclusiva. La maquiladora de exportación se 
ha ido expandiendo como sistema de producción, y en muchos lugares constituye el eje principal de la economía (Villegas et al. 1997).

La ausencia de una organización legitimada que garantice el desempeño del trabajo en condiciones adecuadas, además del proceso de producción característico de las maquilas, es decir, jornadas prolongadas de trabajo, tareas monótonas, presión y competitividad, etcétera, produce a su vez un patrón de desgaste particular, cuya reproducción ${ }^{2}$ es difícil potenciar debido a las condiciones de vida de la población que en ellas labora; a corto plazo este desgaste paulatino se agudiza, y se refleja en la tasa de renuncias o de bajas por motivos de salud, lo que resulta en una rotación elevada de personal (Márquez y Romero 1988; Guendelman et al. 1999). Ante este panorama, la evaluación de la salud de los obreros de las maquilas debe ser una tarea permanente y una prioridad política impostergable, que implica una reconceptualización del riesgo laboral en el sentido que lo señala Denman (1994), y la incorporación de una visión amplia del concepto de salud, ${ }^{3}$ de prevención y de las diferentes dimensiones del bienestar (mental, sexual, psicológico y físico), entre lo que se cuenta prevenir la trasmisión del vIH y proveer servicios de salud, incluso el tratamiento antirretroviral a los trabajadores que lo requieran.

Ninguna persona que labore dentro de la maquila puede asegurar su permanencia en ella; en tanto que la organización del trabajo se orienta en particular hacia la productividad, las bajas por enfermedad o ausencias se traducen en despidos, de manera que lo anterior, aunado a la rotación permanente observada en estos lugares, propicia el desplazamiento de la población hacia otros sitios donde potencialmente pueda ser empleada. De esta manera, los riesgos para la salud propios del lugar de trabajo comienzan a articularse con las condiciones estructurales de vida de los empleados, dan como resultado un contexto de vulnerabilidad propicio para el desarrollo de enfermedades como el VIH/sida.

\section{Movilidad y vulnerabilidad}

Una gran proporción del personal de las maquiladoras es inmigrante, proviene de otras regiones y estados de la república, esta condición de movilidad disminuye el contacto con sus redes sociales, y por lo tanto hace más

\footnotetext{
2 Entendida como la recuperación de lo perdido en el desgaste (Márquez y Romero 1988).

3 No se limita a los accidentes en el trabajo, ni a reportar las causas de consulta más frecuente en este ambiente.
} 
precarias sus condiciones de vida, más por la falta de apoyos afectivos y sociales, que por el factor económico. En el estudio realizado por Bronfman et al. (1995), se reporta que los hábitos sexuales de los hombres mexicanos que emigraron a Estados Unidos cambiaron, debido a que percibieron una mayor "oportunidad", apertura y permisividad para desplegar algún tipo de comportamiento sexual en dicho país, en comparación con el imperante en México; además, fue un medio para satisfacer necesidades afectivas además de sexuales. Muchos de estos cambios, como tener más parejas sexuales, tener sexo con trabajadoras sexuales y con otros hombres (a menudo en combinación con el consumo del alcohol), incrementan las posibilidades de infectarse con el VIH. Magis et al. (2004) afirman que emigrantes internacionales habían tenido más parejas sexuales durante el último año, utilizaron el condón con mayor frecuencia durante su última relación sexual y usaron medicinas inyectadas, así como drogas, en mayor proporción. Estudios recientes con mujeres emigrantes no documentadas revelan que, en el contexto mesoamericano, 60 por ciento de ellas tiene algún tipo de relación sexual durante el viaje, que puede variar desde sexo a cambio de protección o a precio bajo o bien de un guía, hasta violación, sin embargo las condiciones de sometimiento en las que ocurre vulneran a las mujeres a la infección por ITS/VIH, al imposibilitar la negociación del uso del condón (Caballero et al. 2002). Además, en varios estudios, la emigración masculina se ha identificado como una fuente de posible riesgo de contraer el VIH para las parejas femeninas que se quedan (Bronfman et al. 1995; Ortíz-Mondragón et al. 2000; Hirsch et al. 2002; Kendall y Pérez 2004).

Es importante destacar que los comportamientos que hacen vulnerables a las personas a la infección por vIH, por lo general no son por falta de conocimiento; un hallazgo común en las investigaciones con poblaciones móviles en Mesoamérica es que a pesar de que un alto porcentaje sabe que existe el sida y conoce algunas formas de trasmisión y prevención del VIH, reportan comportamiento frecuente de alto riesgo, eso indica que la vulnerabilidad es resultado de un contexto social de vida particular (Bronfman et al. 1995; Madrigal 1998, 1998a). En este mismo sentido apuntan los resultados del estudio de Salgado de Snyder et al. (1996), quienes reportan que 90 por ciento de mujeres mexicanas rurales, parejas de migrantes, manifiestan haber oído del VIH y la mayoría percibe que podría estar en riesgo de infección, pero sólo 5 por ciento de ellas reportó usar siempre el condón. Además, incluso en un mismo contexto general de vida, los hombres y las mujeres se exponen a riesgos diferentes en virtud de su condición de género. De tal forma que esta identidad cultural, construida alrededor de los sexos, permite entender el sometimiento de las mujeres como expresión de las relaciones de poder, que a lo largo de la historia han definido su subjeti- 
vidad, o bien la dificultad de reconocer la práctica homosexual y su riesgo, a partir de una identidad definida sobre la base de la heterosexualidad.

Aunque sin duda se trata de una labor conjunta y transdisciplinaria, una de las personas clave para emprender acciones encaminadas a detener la expansión y efectos de la epidemia de VIH/sida en el lugar de trabajo es sin duda el empleador, en tanto que puede facilitar las condiciones necesarias, en tiempo y lugar, para promover estas acciones; además de ser el enlace entre la empresa y los servicios de salud, tiene poder de gestión para establecer convenios sindicales y leyes laborales que protejan los derechos de los trabajadores, así como los términos de su contratación y la capacidad de propiciar un ambiente laboral saludable; para que dicho actor se involucre en las acciones, debe primero reconocer la vulnerabilidad ante el VIH de la población empleada, y comprender en qué sentido la empresa debería jugar un papel social importante.

La información que a continuación se presenta es el resultado de un estudio diseñado para avanzar en la comprensión de los problemas relacionados con la vulnerabilidad, derechos humanos, estigma y discriminación prevalecientes en un ambiente laboral concreto, como es la industria de la maquila, con el objetivo de explorar las actitudes de los empleadores frente al VIH, y verificar la existencia de acciones y programas actuales para enfrentar la epidemia en este ámbito.

\section{Metodología}

El estudio se realizó en Puebla, entidad mexicana elegida por tener la mayor cantidad de personas empleadas en la industria maquiladora durante 2000 (37 600) (Vargas 2004). Las zonas rurales del estado se caracterizan por su alta marginación y emigración, y por los índices bajos de salud sexual y reproductiva; en 1994 las mujeres entre 15 y 49 años tenían 4.3 hijos en promedio, uno de los más altos entre las entidades federativas (el nacional era de 3.5) (Instituto Nacional de Estadística, Geografía e Informática, INEGI 1994), aunque estas cifras han disminuido en todo el territorio nacional, se sigue manteniendo entre los más altos; en 2007 se registró un promedio de 2.3 (media nacional 2.1) (INEGI 2007). La industria maquiladora en la región emplea también una alta proporción de población emigrante. Además, Puebla es el estado con la razón más cercana de casos de sida entre hombres y mujeres: 3:1, en comparación con el resto del país, donde hay una mujer con sida por cada seis hombres (Magis et al. 2000). 
El desarrollo económico de un estado no siempre es homogéneo, con el fin de abarcar la diversidad, se trabajó en tres regiones de Puebla: norte (municipio de Teziutlán), centro (ciudad de Puebla) y sureste (municipio de Tehuacán); donde la maquila ha tenido una gran expansión, y se ubican grandes industrias diferenciadas por tareas, que forman parte del proceso de la producción. En la región norte y sureste hay un predominio de maquiladoras del vestido, mientras que en la del centro está más diversificada (automotriz, de cartón o de pasta).

\section{Procedimiento}

Se diseñó un cuestionario para empleadores (gerentes general y de recursos humanos y médico de planta), que exploró el perfil de la maquiladora (producción, inversión, tradición), el de las mujeres que laboran en ella según reporte del informante, cobertura de servicios de salud, existencia de programas de prevención y atención a VIH en el lugar de trabajo, así como la percepción del encargado acerca de la vulnerabilidad de sus empleadas a la infección por VIH. Para elaborar el instrumento y realizar el análisis de los resultados, se tomaron como base los indicadores establecidos por ONUSIDA, para medir la implementación de los acuerdos del UNGASS dentro de sitios de trabajo. Se aplicaron 22 cuestionarios.

Se identificaron seis informantes clave, entre académicos con algún trabajo de investigación relacionado; activistas de ONG o miembros de un grupo, cuyas acciones se centraran en la problemática de las maquiladoras y personal activo del sector salud, o con alguna responsabilidad clínica o administrativa hacia el personal de las maquilas. A dichas personas se les aplicó una entrevista semiestructurada (Vela 2001; Kvale 1996), cuya guía fue diseñada sobre los mismos aspectos que contemplaba el cuestionario. A todos se les solicitó la firma de una carta de consentimiento informado antes de contestar el cuestionario o celebrar la entrevista.

Como tercer recurso metodológico, se llevó a cabo una práctica de observación no participante (Woods 1987), sin un tiempo específico ya que se realizó de manera intermitente, y durante ella los trabajadores de campo obtuvieron información orientada a la descripción y comprensión del contexto de estas industrias (se elaboró una guía de observación que incluyó verificación de programas y acciones, medidas de seguridad y ambiente y contexto de vida de las mujeres). El foco de observación fue el lugar de trabajo y vivienda de las trabajadoras y, cuando fue posible, se observó también el espacio de la propia maquila. 
Todas las técnicas fueron aplicadas por tres antropólogos capacitados, que cubrieron cada región. Los resultados que se presentan a continuación son producto de estas tres fuentes de información: cuestionario, entrevista y observación.

\section{Resultados}

Perfil de las maquiladoras de las comunidades visitadas

Las 22 maquiladoras estudiadas variaron en tamaño, se encontró desde el taller de cinco personas hasta la empresa con mil empleados, tanto hombres como mujeres, cuyo total, estimado por los gerentes, fue de 2840 .

Todas las de Tehuacán y Tezuitlán incluidas en el estudio $(n=15)$ eran textiles, dedicadas a la fábrica de ropa, y todos los gerentes identificaron su tipo de inversión como nacional, aunque varios reconocieron que exportaban su mercancía a Estados Unidos; esta información se confirma con el testimonio de los informantes clave. La principal diferencia entre las maquiladoras de Tezuitlán y Tehuacán fue su tiempo promedio de operación; en Tehuacán era de 6.8 años, mientras que, con la exclusión de una de 13 años, el de Tezuitlán era de 2.3. Lo anterior contrasta con lo manifestado por una empleada de la supervisión federal de educación primaria, quien indicó que el desarrollo de las maquilas en Teziutlán data de la década de 1970, y que el auge de dichas empresas comenzó hace diez años y se mantiene a la fecha.

En la ciudad de Puebla hubo más variación en el tipo de industria e inversión, en cuanto a la primera se encontraron de productos químicos, automotrices y textiles y en lo relativo al segundo factor, hubo tres nacionales, tres de inversión internacional y una de capital mixto; su promedio de operación fue de nueve años.

Un hallazgo interesante es que los individuos que respondieron al cuestionario en general eran originarios del lugar, con cierta estabilidad en el empleo como gerentes, en virtud de que la mayoría ocupaba su puesto casi el tiempo que la maquiladora llevaba operando.

A pesar de que todas las empresas visitadas declararon tener permiso legal para operar, fue posible observar talleres de confección detrás de fachadas que aludían a espacios dedicados a otro fin como "almacén de mezclilla" o "tortillería”. Lo anterior, si bien no se contradice, prueba la existencia de maquiladoras que no cuentan con un registro legal y operan de forma clandestina, con las consecuentes irregularidades que pueden suponerse para sus empleados, como falta de seguridad social u otras prestaciones. La maquila domés- 
tica, ${ }^{4}$ más que la de exportación, es la que se presta más a esta situación de ilegalidad “ $[\ldots]$ en esas maquilas, de todas las situaciones peores, de clandestinidad, se dan en el universo de la maquila para el mercado doméstico, en la maquila clandestina [...]” (informante clave, Tehuacán).

Este tipo de talleres clandestinos evaden sobre todo las responsabilidades fiscales y municipales a las que están comprometidas las maquiladoras legales, y en el caso de las de exportación no pagan aranceles o evitan el pago de servicios como uso de suelo, agua y luz; no tienen salarios fijos para sus trabajadores e incurren en relaciones laborales irregulares. Muchas de ellas operan con una plantilla de obreros cuya gran mayoría es menor de edad, a pesar de que en 1998 se implementaron códigos específicos para regularizar esta situación (informante clave, Tehuacán).

Los informantes clave de Tehuacán mencionaron que desde 2001 ha crecido el mercado de exportación en la región, lo que ha generado un proceso de reconversión del sector y se refirieron también a ciertas irregularidades en las que incurren algunas empresas, pues pese a existir una ley laboral que las obliga a tener un contrato colectivo de trabajo, éstos suelen ser indeterminados, hay maquilas que cambian de razón social constantemente como estrategia para evitar la reanudación de contratos, otras se establecen uno o dos semestres en un lugar y se mudan a otro barrio, y cambian su apariencia. El aburrimiento y el cansancio son otros mecanismos de algunas empresas para obligar a sus trabajadores a renunciar antes de tener que elaborar contratos nuevos. En el trabajo a domicilio y en las maquilas domésticas esta situación es aún más irregular, aquí incluso no se llega a pagar el salario mínimo, es frecuente que el sueldo sea menor al establecido por ley (notas de campo, informante clave, Tehuacán).

Gran cantidad de estas industrias, según declaración de los informantes clave, funcionan con el régimen de subcontratación; trabajan para las grandes maquiladoras para elaborar una parte específica del proceso productivo, cuando no tienen la capacidad de fabricar las cantidades que el cliente exige. Otras carecen de un registro legal y sólo tienen permisos renovables, esta condición les permite desvincularse de toda responsabilidad frente a sus empleados, quienes trabajan por "periodos de prueba" en lugar de contrato, rotan de labor dentro del proceso productivo, y así cada dos o tres meses inician otro periodo de prueba en una actividad diferente pero dentro de la misma maquiladora. Esta y otras estrategias empresariales evitan proveer

\footnotetext{
${ }^{4}$ No necesariamente es de carácter familiar, puede haber 15 o 20 máquinas y hasta 200 trabajadores (informante clave, Comisión de Derechos Humanos y Laborales del Valle de Tehuacán).
} 
prestaciones a los trabajadores, como seguro médico, aguinaldo o vacaciones, como lo refiere el testimonio siguiente:

[...] en una semana el empleador dijo [refiriéndose a un caso concreto] - pues ahora los salarios van a ser así- y les bajó 50 por ciento, entonces, bajarte el salario es en contra de la ley, pero no lo hacen con uno, lo hicieron con más de mil trabajadores, entonces es una forma que la gente tenga que salirse porque no va a poder sobrevivir con eso ¿no? Las empresas, generalmente hacen prácticas de aburrir a la gente, bajarles salarios o de plano despedirlos por cualquier tontería injustificada. (Informante clave, Tehuacán).

\section{Perfil de las mujeres que trabajan en la maquiladora}

Alrededor de 60 por ciento de las mujeres empleadas de las maquiladoras textiles de Tehuacán y Tezuitlán tiene un perfil semejante, están en edad reproductiva (20 a 30 años), con educación primaria o al menos con habilidades para leer y escribir; solteras, en unión libre o casadas, un dato remarcado constantemente fue que muchas de ellas eran madres solteras. Una gran cantidad proviene de pueblos cercanos a Puebla y también de Oaxaca, Chiapas y Veracruz.

Lo anterior fue confirmado por los informantes clave, quienes también comentaron que 80 por ciento de la población que trabaja en las maquilas es indígena, de los grupos mazateco, mixteco y popoloca; 50 por ciento estaba constituido por inmigrantes de barrios y comunidades vecinas e incluso de otros estados y países de Centroamérica, y que la maquiladora es el único marco de referencia para esta población.

Sin embargo, en cuanto a la edad, pese a que la mayoría de los empleadores declararon no contratar a menores de edad, a través de la observación se identificó a niñas trabajadoras (notas de campo).

Puesto que el perfil de las maquiladoras en Puebla es diferente respecto al de Tehuacán y Teziutlán, no fue sorprendente identificar que también el de las empleadas era distinto. La proporción de la fuerza de trabajo femenina variaba entre 10 y 80 por ciento según los empleadores, quienes dijeron que las mujeres tenían mayor nivel de educación, la mayoría cuenta con secundaria. Sin embargo, varios de los encargados indicaban que una gran proporción de las empleadas eran madres solteras, y expresaban una preferencia hacia la contratación de mujeres con hijos por considerarlas más estables como trabajadoras.

Los informantes refieren que hace 15 años la población de la maquila estaba constituida en particular por mujeres, ubicadas sobre todo en tareas 
de lavandería, cocina o costura (consideradas propias de su función de género), pero esta situación ha ido cambiando debido sobre todo a la crisis y a la emigración, de tal forma que el porcentaje de participación de los hombres ha ido en aumento.

\section{Servicios de salud para las trabajadoras}

De los encargados, 95 por ciento manifestó que las trabajadoras de las fábricas estaban aseguradas en el Instituto Mexicano del Seguro Social (IMSS). El gerente general de una en Tehuacán expresó que las mujeres tienen acceso a servicio médico privado externo, y de ser necesario se canalizan a un hospital particular. Se dijo tener servicios de salud dentro de la maquiladora en 41 por ciento de los casos, y en el mismo porcentaje se afirmó que pedín exámenes de salud a las aspirantes antes de emplearlas o de manera periódica. En Puebla, tres gerentes dijeron que solicitaban estudios antes de la contratación; en dos fábricas además de uno de salud general, pedían pruebas de embarazo, vista, ITS y viH. Los exámenes periódicos mencionados fueron de la vista, Papanicolaou y cáncer de mama.

Esta información contrasta con los registros realizados en la práctica de observación, en los que se señala que son pocas las maquiladoras con servicio médico propio, en el mejor de los casos se identifica la presencia de una enfermera o un médico de medio tiempo. En Teziutlán, el informante, profesional de la salud con cargo directivo, comentó:

$[\ldots]$ yo creo que no [cuentan las empresas con servicios médicos para atender emergencias y dar primeros auxilios], [...] posiblemente botiquín, primeros auxilios sí, es también de ley tenerlo, es de protección civil una recomendación importante, pero que yo tenga la certeza de que tienen un médico, que tienen un lugar específico para atender pacientes, me parece que no, eso sí ninguna maquila creo que lo tenga [...] (informante clave, Teziutlán).

Ofrecer seguridad social a los empleados parece depender de las características de la maquiladora, las medianas y grandes fábricas suelen tenerlo, aunque hay empresas que no pagan las cuotas correspondientes y pierden este beneficio.

En el caso de Tehuacán, fue entrevistado un activista por los derechos de los trabajadores de las maquilas, quien comentó que a pesar de la obligación de las empresas de ofrecer la seguridad social, no se hacía efectiva en la práctica, mucha gente en la maquila no la tiene y las empresas no pagan las cuotas. Varios empleadores prefieren dar vales para que las mujeres acudan a 
médicos privados antes que asegurarlas en el IMSS, como correspondería por ley, ante accidentes laborales, la empresa cubre los gastos de un servicio particular a fin de persuadirlas para que no vayan al IMSS, donde pueden enterarse de que el patrón no ha pagado la cuota y evitar así una demanda

[...] y eso es muy común, o sea, hay plantas que tienen doctores [particulares], o sea tienen que tener enfermería y muchas hacen eso, para evitar que la gente vaya a recibir los servicios de salud [...] se accidentan y los llevan [al seguro social], y no les pueden dar el servicio porque no están dados de alta o porque el patrón tiene tres meses que no se pone al corriente de las cuotas [...] (informante clave, Tehuacán).

En cuanto a la prueba de gravidez, comenta que antes se solicitaba con mayor frecuencia e incluso con carácter de obligatoriedad a las mujeres, pero esta situación ha ido cambiando y ahora muchas empresas se conforman con explorarlo de manera verbal. De cualquier modo, dijo, no hay prestaciones por concepto de maternidad.

\section{Programas de prevención contra el viH en el lugar de trabajo}

En el cuadro 1 se observan los resultados en términos de programas para prevenir el contagio de VIH, y reducir el estigma y discriminación asociados con el viH/sida dentro del lugar de trabajo:

\section{Cuadro 1}

Programas de prevención

\begin{tabular}{|l|c|c|}
\hline Acción & Sí (porcentaje) & No (porcentaje) \\
\hline Favorecen el reclutamiento de Pvvs & $1(5)$ & $21(95)$ \\
Campañas/educación anti-discriminatorias & $1(5)$ & $21(95)$ \\
Educación sobre viH/sida & $6(27)$ & $16(73)$ \\
Distribución de condones & $2(9)$ & $20(91)$ \\
Consejería voluntaria y prueba del viH & - & $22(100)$ \\
Servicio para diagnóstico y tratamientos de rTs* & - & $22(100)$ \\
\hline
\end{tabular}

Fuente: proyecto de investigación: “Trabajadoras de la maquila y vIH/sida”. Investigadora responsable: Blanca E. Pelcastre Villafuerte; colaboradores: Mirka Joan Negroni Belén y Tamil Kendall $(10 / 2003$ a 12/2004).

* Ningún empleador manifestó tener programas de diagnóstico y tratamiento de ITS, sin embargo una fábrica en Puebla y tres en Tehuacán reportaron que el Papanicolaou es uno de los exámenes médicos solicitados dentro de la empresa. 
Con excepción de educación puntual básica sobre VIH (pláticas impartidas por ONG) en una minoría de sitios de trabajo (27 por ciento), el panorama de prevención y reducción de estigma y discriminación en este ámbito es mínimo. Sólo una empleadora resaltó una experiencia positiva de educación sobre cuestiones de sexualidad y planificación familiar, ella refirió un incremento de conocimientos en los trabajadores y un mayor interés entre ellos; cuatro de los empleadores mencionaron que alguna vez el IMSS donó condones y otros dos manifestaron su disposición a distribuirlos si el IMSS u otra instancia se los proporcionaba. Sólo uno reconoció haber tenido malas experiencias distribuyendo condones, como burlas o uso de ellos como globos. En ninguno de los testimonios de la población trabajadora, recogidos durante la práctica de observación, se mencionó que se impartieran pláticas sobre sexualidad o se llevaran a cabo acciones como repartir condones.

Los empleadores identificaron como barreras para implementar programas de educación de salud en general (no específicamente en VIH), que se realizaran en el tiempo libre del personal, pues éste prefería descansar, sin embargo no señalaron la disposición de hacerlo durante las jornadas laborales; algunos dudaron de la receptividad de los trabajadores a este tipo de proyecto, por desconfianza o falta de educación.

El activista señaló la existencia de un programa municipal de mujeres que abordaba sobre todo el problema de la violencia doméstica, sin embargo opinó que sus acciones eran limitadas, habían realizado un foro y daban talleres, pero el contacto directo con la gente era muy poco, las mujeres debían acercarse al programa y no a la inversa; de manera que remarcó la falta de un mayor vínculo con la sociedad, pues en este sentido no se podían identificar avances. Reconoció que en la región se presentan problemas de vIH/sida, violencia y otros que deben enfrentarse.

El profesional de la salud mencionó que si bien se había proporcionado información relativa a la prevención del vIH/sida en algunas maquiladoras, por parte de las instituciones de salud a solicitud de los empleadores, no había sido suficiente. Desde su punto de vista, para implementar campañas de prevención del viH hacen falta insumos, preservativos más que folletos para trabajar, pues percibe una apertura para que las empresas acepten este tipo de campañas entre su personal.

\section{Beneficios para las PVvs}

Uno de los empleadores manifestó que no contrataría a alguien que viviera con VIH/sida, debido a que "[...] el IMSS multa a las empresas cuyos empleados padecen enfermedades costosas”. 
El profesional de la salud entrevistado comentó que no conocía casos en los que las personas no hayan sido contratadas por tener viH/sida, e incluso que se les exigiera la prueba. La Secretaría de Salud (SSA), dijo, es la encargada de atender a las Pvvs, sin embargo ha habido personas que han tenido que emigrar a otros lugares para su tratamiento, cuando requiere ser más especializado.

\section{Percepción sobre la vulnerabilidad de las empleadas}

La mayoría de los empleadores, 16 (73 por ciento), señaló que sus trabajadoras son vulnerables a la infección por VIH, 6 (27) expresaron que no. Los dos médicos de planta entrevistados declararon que las mujeres no son vulnerables a la infección por viH, porque "la población es muy sana, la mayoría casada”, y que nunca habían recibido alguna mujer golpeada en su hogar, así "que todas tienen buenas vidas". Cabe mencionar que uno de los médicos constató que no se debe favorecer el reclutamiento de pvvs porque significaría someterlas a trabajos poco benéficos para ellas, por el rigor de la actividad y el horario. También dijo que se puede identificar a simple vista a alguien infectado con VIH por la palidez del rostro, la pérdida de peso y porque sufre desmayos.

Desde el punto de vista de un informante clave, el nivel de escolaridad (de no más de secundaria) y la cultura de los y las trabajadoras influyen en la baja percepción de riesgo que asumen para ellas mismas

[...] ese mismo nivel de cultura hace que no se den cuenta del riesgo que corren, que no tomen las precauciones necesarias, que se dejen llevar por el instinto que todos tenemos, y sin importarles ninguna consecuencia, entonces, yo diría que son personas altamente vulnerables, que tal vez sean nuestros principales puntos de atención, de infección en el municipio $[\ldots]$ (informante clave, Teziutlán).

Los informantes clave profesionales de la salud señalaron que había mucha promiscuidad y relaciones sin responsabilidad en esta población, lo que generaba un alto porcentaje de madres solteras jóvenes en la región, dijo haber recibido denuncias de casos de actos sexuales en el baño del mismo centro de trabajo.

\section{Estigma y discriminación}

Ellos también manifestaron que la región era un foco de infección importante, y que los trabajadores de la maquila corren un alto riesgo de contra- 
er el vIH. A pesar de que la información existente es limitada, opinan que el personal está informado, pero es incongruente con sus acciones; indicaron que la mayoría de las personas que mueren por vIH/sida son mujeres, en una proporción de $3: 1,^{5}$ e identificaron comportamientos que califican como culturalmente inaceptables ("las mujeres abren fácilmente las piernas”) detrás de estas estadísticas.

El discurso estigmatizado de riesgo asociado con la actividad sexual fue el más común. Las frases siguientes ejemplifican este discurso: “porque está de moda que las mujeres sean liberales y ya no se espantan tan fácil como antes". "Muchas veces la gente no hace caso de lo que dice el médico y corren el riesgo de tener sida si no utilizan condón o son promiscuos" o "llega gente que tiene tres hijos de tres padres diferentes".

\section{Estigma y discriminación hacia las Pvvs en el sitio de trabajo}

Los empleadores dijeron que había mucho rechazo hacia las Pvvs por parte de los y las trabajadoras y por eso no las contratarían, para no provocar miedo en los otros empleadores; uno de ellos llegó a decir que tampoco lo haría debido a que perjudicaría la imagen de la empresa, porque sería foco de infección. Más común fue la negación de contratar a una Pvvs debido al alto riesgo que ello implicaba, ya que utilizan instrumentos punzo cortantes (agujas, navajas). Al respecto, un informante clave profesional de la salud comentó que las personas que viven con VIH son rechazadas porque la población le teme sobre todo al contagio; desde su punto de vista, la falta de conocimiento es la principal causa de este comportamiento

[...] la falta de información, la falta de cultura al respecto, hace que uno le, no uno, me pongo en el lugar de las personas, que la población les tenga temor [a las Pvvs], que les tenga cierto rechazo a un posible contagio, repito, por la falta de conocimiento, porque uno sabe las vías de contagio, son directas, jamás va a ser por otros medios, tal vez la falta de conocimientos sí ha generado que algunos sectores sí las traten de aislar, separarles, tenerles un poquito de rechazo como le comentaba, desgraciadamente sí falta un poquito más promover esa cultura $[\ldots]$ aquí en el municipio[...]. (Informante clave, Teziutlán).

\footnotetext{
${ }^{5}$ Es importante señalar que la percepción expresada por el informante contradice las estadísticas, que reportan tres casos de sida masculinos por cada caso femenino en el estado de Puebla.
} 
En términos generales, empleadores e informantes clave percibieron una alta probabilidad de discriminación por parte de los y las trabajadoras de las maquilas, hacia Pvvs. El activista reconoció que ninguna persona debería ser rechazada por tener VIH/sida o por razones de género y que en este sentido, el papel del gobierno es más bien pasivo, pues no actúa a menos que haya una denuncia de por medio, cosa que rara vez se presenta.

El silencio, el estigma y la discriminación son barreras claras para enfrentar la epidemia del VIH/sida en el ambiente laboral, desde la perspectiva de las personas que emplean a hombres y mujeres en sus empresas.

\section{Discusión}

En términos generales, se puede decir que el mercado laboral en Tezuitlán es más precario con respecto al de Tehuacán, en tanto que en este último aún no se consolida esta rama. A pesar de las variaciones y las tendencias, hoy en día la participación de mano de obra femenina sigue siendo importante en este contexto, por tanto, se considera que el perfil sociodemográfico de las mujeres que trabajan en la maquiladora textil nacional, descrito en términos de nivel de escolaridad bajo, en edad reproductiva, además de la condición de emigrante son factores que pueden contribuir a la vulnerabilidad de esta población a la infección por viH, así como a un estado de salud sexual y reproductiva en general pobre. Sin embargo, este no es el único factor, las condiciones de irregularidad, subcontratación y clandestinidad en las que operan muchas maquiladoras, reportadas ya en otros estudios como el de Barrios y Santiago (2004), despojan a las mujeres de su derecho y poder de negociación generando condiciones de inseguridad que pueden repercutir en forma negativa sobre ellas, y aumentar su vulnerabilidad a varias formas de explotación, incluso las que las expongan a la infección. Así, la pobreza y la falta de oportunidades ha sido aprovechada por los dueños de las empresas para hacerse de un ejército de costureras y cortadores, quienes al no encontrar trabajo en otra parte, aceptan condiciones que los vulneran y hacen posible la violación de sus derechos humanos y laborales.

A través del análisis de los resultados fue posible identificar dos discursos confrontados: el institucional o patronal versus el de los trabajadores (en la voz del activista), que en el primer caso asegura la prestación de la seguridad social a la casi totalidad de los y las trabajadoras; en el segundo, niega que ésta se haga efectiva en los hechos. La falta de denuncias al respecto no significa que los y las empleadas cuenten con esta prestación, ni la solicitud verbal de no gravidez significa que se haya superado la discriminación de 
género y alcanzado la equidad, es más, esta última situación continúa denunciándose en algunos estudios (Human Rights Watch 1996, 1998). Aunque en apariencia irreconciliables, estas informaciones permiten remarcar el carácter de obligatoriedad de este servicio con base en una ley laboral que así lo estipula, y reconocer en la Comisión de Derechos Humanos y Laborales del Valle de Tehuacán un recurso potencial a través del cual pueda hacerse efectivo este derecho, a fin de estructurar una red de apoyo institucional que potencie la participación del IMSS y servicios de salud privados, que haga frente a la vulnerabilidad de las mujeres ante el viH. Los indicadores para medir beneficios adicionales para pvvs en términos de acceso a tratamiento (antirretrovirales, farmacéuticos contra infecciones oportunistas y permisos para acudir a citas médicas) deberían ser cubiertos si todas las mujeres tuvieran acceso efectivo a servicios de salud a través del IMSS.

Es clara y alarmante la ausencia de acciones y programas encaminados a la prevención del viH/sida en las maquiladoras visitadas en Puebla, en ocasiones reparten condones, pero no hay seguimiento a estas acciones concretas ni un marco informativo que le dé sentido a la acción. Se reconoce la necesidad de implementar dichos programas, y aprovechar la disposición manifiesta del profesional de la salud, en quien se advierte un recurso potencial en virtud de su reconocimiento de la necesidad de intervenir, y lograr además el apoyo de instituciones como el IMSS.

Se identificaron dos ejes principales del estigma y la discriminación, uno basado en un discurso moral que descalifica las prácticas sexuales de la población, y otro en la disposición a la discriminación hacia las pvvs en el sitio de trabajo. Dichos ejes se manifestaron en que sólo una de las empresas señaló favorecer el reclutamiento de pvvs, la posibilidad de discriminación activa fue mucho más evidente en los comentarios libres de los empleadores. Sin embargo, a pesar del poco conocimiento sobre VIH y la alta discriminación potencial mostrada por los empleadores y prestadores de servicios de salud, resulta positivo que la mayoría de los primeros perciban la vulnerabilidad de sus trabajadoras al $\mathrm{VIH} / \mathrm{sida}$, porque incrementa las posibilidades de sensibilización y fomenta el impulso de programas de prevención ayudando a ello su estabilidad en el puesto, porque los convierte en un blanco estratégico para su implementación. Los comentarios de algunos informantes clave denotaron confusión para identificar la infección por viH y el cuadro clínico asociado al sida, además de reflejar un estereotipo estigmatizante relacionado con la debilidad física de las pvvs. Lo anterior, aunado al juicio moral implícito expresado en el discurso estigmatizado de riesgo y las relaciones asumidas poco fundamentadas, como el hecho de igualar el matrimonio a una condición de bajo riesgo o la admisión de que la violencia no existe por 
el hecho de no haber atendido casos, obstaculiza la prevención y alienta la discriminación hacia las personas que viven con vIH/sida. Esto llama la atención sobre la necesidad de capacitar, en particular a los prestadores de servicios de salud, por ser quienes mantienen un contacto más directo con la población, sobre aspectos tanto médicos como sociales relacionados con el VIH.

Los tres discursos relevantes de los empleadores: el de la culpa sexual, la vulnerabilidad de toda la población (sin identificar especificidades) y, en algunos casos, un entendimiento global de la vulnerabilidad estructural (pobreza, maternidad fuera del matrimonio, edad reproductiva, falta de información y opciones) dan cuenta de las relaciones que establecen estos actores, en un esfuerzo por explicar el riesgo de la población, lo que puede constituir un buen punto de partida para el diseño de una participación.

Cabe señalar que en cuanto a las acciones preventivas, se han reportado intervenciones específicas para poblaciones móviles (Magis et al. 2004), que sin ubicarse concretamente en la industria maquiladora, pueden incluir a su población trabajadora, no obstante, que su repercusión se ha medido en términos de la cobertura que alcanzan más que en los conocimientos o comportamientos. Es necesario trabajar más en este sentido, Kendall y Langer (2006) señalan que en las comunidades de origen de los y las emigrantes la respuesta a la salud de esta población en materia de VIH y sida se caracteriza por acciones aún limitadas, tanto de parte de los gobiernos como de la sociedad civil. Existen diversos programas de promoción de la salud, pero en su mayoría se limitan a la repartición de materiales educativos y pláticas informativas. Las autoras subrayan la importancia de extender el acceso a pruebas diagnósticas de VIH, preservativos y desarrollar vías de comunicación más innovadoras y atractivas para poblaciones móviles en áreas rurales. En el ámbito internacional, existen ejemplos de programas de prevención del VIH con hombres inmigrantes mexicanos o latinos que han provocado cambios de comportamiento; estos programas se caracterizan por combinar medios de comunicación culturalmente apropiados (radionovelas, fotonovelas), diseñados en específico para la población con educación, por parte de pares capacitados (Mishra et al. 2004; Somerville et al. 2006).

\section{A manera de conclusiones}

- La clandestinidad e irregularidades en el estatus legal de las maquiladoras dificulta las acciones conjuntas encaminadas a la prevención y atención del vIH. 
- Hasta la fecha, la intervención en prevención dentro de las maquiladoras en estas zonas ha sido mínima. Está lejana la meta para tener los sitios de trabajo definidos por el UNGASS.

- Se identificó falta de conocimiento sobre viH/sida entre los médicos de planta y el personal de salud entrevistado, además este último reconoció la ausencia de servicios especializados y personal capacitado para atender casos de VIH/sida fuera de la capital del estado.

- Los empleadores identifican el bajo nivel de escolaridad y de interés de las trabajadoras como obstáculos para la implantación de programas de prevención.

- Se identificaron actitudes de estigma y discriminación relacionadas con VIH entre los empleadores e informantes clave entrevistados, además ellos percibían actitudes similares entre los y las trabajadoras. Cualquier programa tendría que ser diseñado cuidadosamente para evitar la posibilidad de que la intervención incrementara el estigma y discriminación hacia las Pvvs.

Dicho lo anterior, se recomienda:

- Considerar la colaboración con el IMSS como vía de acción promisoria.

- Pensar en la afiliación de las trabajadoras al seguro popular, para campañas de promoción.

- Impulsar acciones educativas y programas de cambio de actitudes con personal de salud.

- Planear estrategias de intervención en horarios y lugares diferentes a las maquiladoras, pero sin llegar a ser inaccesibles, en virtud de que no todos los gerentes mostraron disposición para ceder horas de las jornadas laborales para actividades y planes de prevención.

- Vincular programas gubernamentales de manera directa con la población, y no esperar que ésta acuda a ellos.

- Articular las acciones de intervención para prevenir el VIH con otras que legalicen la operación de las maquiladoras, a fin de que esta regularización promueva un trabajo en el marco de los derechos humanos y laborales.

- Acompañar las acciones concretas como el reparto de condones en los centros de trabajo, con tareas de información y seguimiento.

- Diseñar intervenciones integrales, basadas en estrategias exitosas con poblaciones emigrantes en otros contextos, y adecuarlas a las creencias y necesidades de las mujeres trabajadoras.

Los datos del estudio permitieron alcanzar el objetivo planteado, se resaltó la perspectiva cualitativa como una fuente importante a partir de la cual 
es posible identificar ejes para la acción, ya que lo fundamental de esta aproximación es recuperar la experiencia de los actores involucrados e identificar, a partir de sus discursos, elementos relevantes que no sólo se ubican en la acción individual, sino que la trascienden. Así mismo, la estrategia de triangulación a partir del uso de diversas técnicas (Arias 2000) permitió fortalecer la credibilidad del estudio (Rodríguez, Gil y García 1999, 286-287), cuya transferibilidad (Lincoln y Guba 1985) es posible dadas las características que definen la industria maquiladora en el país.

Recibido en marzo de 2007

Revisado en noviembre de 2007

\section{Bibliografía}

Arias, María Mercedes. 2000. La triangulación metodológica: sus principios, alcances y limitaciones. En Paradigmas y diseños de la investigación cualitativa en salud, compilado por Francisco Mercado, Denise Gestaldo y Carlos Calderón, 481-499. Guadalajara: Universidad de Guadalajara.

Bronfman, M., A. Amuchástegui, R. M. Martina, N. Minello, M. Rivas y G. Rodríguez. 1995. Sida en México: migración, adolescencia y género. México: Consejo Nacional para la Prevención y Control del viH/sida (CONASIDA) e Información Profesional Especializada.

Barrios Hernández, Martín A. y Rodrigo Santiago Hernández. 2004. Tehuacán: del calzón de manta a los blue jeans. La nueva industria del vestido en México, los trabajadores y las comunidades indígenas. Puebla: Comisión de Derechos Humanos y Laborales del Valle de Tehuacán, A. C.-Red de Solidaridad de la Maquila.

Caballero, M., A. Dreser, R. Leyva, C. Rueda y M. Bronfman. 2002. Migration, Gender and HIV/AIDS in Central America and Mexico. En Advocacy and Policy. XIV AIDS International Conference, 263-267. Bologna: Monduzzi Editore.

Denman, Catalina. 1994. Madres y maquiladoras en Nogales, Sonora. En Nuevos textos y renovados pretextos, coordinado por Vania Salles y Elsie McPhail, 277316. México: El Colegio de México-PIEM.

Guendelman, Sylvia; Stevens Samuels y Martha Ramírez. 1999. Relación entre salud y renuncia al empleo en trabajadoras de la industria maquiladora electrónica de Tijuana. Salud Pública de México 41 (4): 286-296. 
Hirsch, Jennifer; Jennifer Higgins, Margaret E. Bentley y Constance A. Nathanson. 2002. The Social Constructions of Sexuality: Marital Infidelity and Sexually Transmitted Disease-HIv Risk in a Mexican Migrant Community. American Journal of Public Health 92 (8): 1227-1237.

Human Rights Watch. 1998. A Job of your Rights: Continued Sex Discrimination in Mexico's Maquiladora Sector. Nueva York: Human Rights Watch.

1996. Sin garantías. Discriminación sexual en el sector de maquiladoras de México. Nueva York: Human Rights Watch.

International Labour Organization-AIDS. 2004. Why AIDS is a Workplace Issue? http://www.ilo.org/public/english/protection/trav/aids/why/ index.htm (9 de marzo de 2005).

INEGI. 2007. Tasa global de fecundidad por entidad federativa 2000 a 2007. http://www.inegi. gob.mx/est/contenidos/espanol/rutinas/ept.asp?t= mpob17\&c=3194 (9 de octubre de 2007).

1994. Encuesta nacional de la dinámica demográfica 1992. Principales resultados. México: INEGI.

Kendall, Tamil y Ana Langer. 2006. VIH/sida y migración México-Estados Unidos: evidencias para enfocar la prevención. En Los mexicanos de aquí y de allá: problemas comunes. Memorias del segundo foro de reflexión binacional, 139-154. México: Senado de la República LIx Legislatura-Fundación Solidaridad Mexicano Americano.

Kendall, Tamil e Hilda Pérez. 2004. Las mujeres mexicanas VIH positivas hablan: necesidades y apoyos en el ámbito médico, familiar y comunitario. México: Colectivo Sol.

Kvale, S. 1996. Interviews. An Introduction to Qualitative Research Interviewing. Londres: Sage.

Lincoln, Yvonna y Egon Guba. 1985. Naturalistic Inquiry. Beverly Hills: Sage Publications.

Madrigal, Johnny. 1998. En las trincheras de la confianza. Una encuesta sobre el condón en las trabajadoras comerciales del sexo de América Central. San José: Instituto Latinoamericano y del Caribe de Planificación Económica y Social (ILPES). 
1998a. Al vaivén de un cabezal. Un estudio sobre los traileros en América Central y su relación con el sida. San José: ILPES.

Magis, Carlos; Cecilia Gayet, Mirka Negroni, René Leyva, Enrique Bravo, Patricia Uribe y Mario Bronfman. 2004. Migration and AIDS in Mexico. An Overview Based on Recent Evidence. Journal of Acquired Immune Deficiency Syndromes 37 (suplemento 4): S215-S226.

Magis, Carlos; Enrique Bravo y Pilar Rivera. 2000. El sida en México en el año 2000. En La respuesta mexicana al sida: mejores prácticas, compilado por Patricia Uribe y Carlos Magis, 13-22. México: CONASIDA.

Márquez, Margarita y Josefina Romero. 1988. El desgaste en las obreras de la maquila eléctrico-electrónica. Salud Problema 14: 9-24.

Mishra, S. I., F. Sanudo, R. F. Conner. 2004. Collaborative Research toward HIV Prevention among Migrant Farmworkers. En Preventing AIDS: Community Science Collaborations, editado por B. P. Bowser, S. I. Mishra, C. J. Reback y G. F. Lemp, 61-95. Nueva York: The Haworth Press.

Naciones Unidas. 2001. Declaración de compromiso en la lucha contra el sida. Periodo extraordinario de sesiones de la Asamblea General de las Naciones Unidas sobre el viH/sida.

OIT. 2001. Repertorio de recomendaciones prácticas de la Organización Internacional del Trabajo sobre el viH/sida en el mundo del trabajo. http://www.ilo.org/public/english/ protection/trav/aids/code/languages/hiva4s.pdf (9 de marzo de 2005).

ONUSIDA. 2002a. Campaña mundial contra el sida 2002-2003. Marco conceptual y base para la acción: estigma y discriminación relacionados con el viH/sida. http://www.e-alliance.ch/media/media-4304.pdf (3 de octubre de 2007).

2002b. Mantener la promesa: resumen de la declaración de compromiso en la lucha contra el sida. Ginebra: ONUSIDA.

Ortíz-Mondragón, Raúl; Carlos Magis-Rodríguez, Martha Loya-Sepúlveda, Jarumi Silva-Bustillos y Patricia Uribe-Zúñiga. 2000. Riesgo de VIH/sida en mujeres parejas de migrantes temporales a los Estados Unidos. 
Ponencia presentada en la II Conferencia de cooperación técnica horizontal en VIH/sida y ETS 2000, Sao Paulo.

Rodríguez, Gregorio; Javier Gil y Eduardo García. 1999. Metodología de la investigación cualitativa. Málaga: Ediciones Aljibe.

Salgado de Snyder, Velia Nelly; M. J. Díaz Pérez y Margarita Maldonado. 1996. AIDS: Risk Behaviors among Rural Mexican Women Married to Migrant Workers in the United States. AIDS Education and Prevention (8): 134-142.

Sommerville, G. G., S. Diaz, S. Davis, K. D. Coleman y S. Taveras. 2006. Adapting the Popular Opinion Leader Intervention for Latino Young Migrant Men who have Sex with Men. AIDs Education and Prevention 18 (6): 573-582.

UNAIDS. 2007. Fact Sheet. http://www.unaids.org/en/Regions_Countries/ Regions/default.asp (28 de febrero de 2007).

2006. Global Report Annex 3: Country Progress Indicators. http://data.unaids.org/pub/GlobalReport/2006/2006_GR_ANN3_en. pdf (2 de octubre de 2007).

Vargas, Lucinda. 2004. Maquiladoras: Still Growing. http://www.maquilaportal.com/public/artic/artic96e.htm (31 de mayo de 2004).

Vela, Fortino. 2001. Un acto metodológico básico de la investigación social: la entrevista cualitativa. En Observar, escuchar y comprender. Sobre la tradición cualitativa en la investigación social, coordinado por María Luisa Tarrés, 63-95. México: Facultad Latinoamericana de Ciencias Sociales-El Colegio de México.

Villegas, Jorge; Mariano Noriega, Susana Martínez y Sandra Martínez. 1997. Trabajo y salud en la industria maquiladora mexicana: una tendencia dominante en el neoliberalismo dominado. Caderno de Saúde Pública 13 (suplemento 2):123-134.

Woods, Peter. 1987. La escuela por dentro: la etnografía en la investigación educativa. Barcelona: Paidós. 
